A microfluidic nanoliter mixer with optimized grooved structures driven by capillary pumping

This article has been downloaded from IOPscience. Please scroll down to see the full text article.

2006 J. Micromech. Microeng. 161358

(http://iopscience.iop.org/0960-1317/16/7/033)

The Table of Contents and more related content is available

Download details:

IP Address: 140.112.113.225

The article was downloaded on 19/12/2008 at 05:55

Please note that terms and conditions apply. 


\title{
A microfluidic nanoliter mixer with optimized grooved structures driven by capillary pumping
}

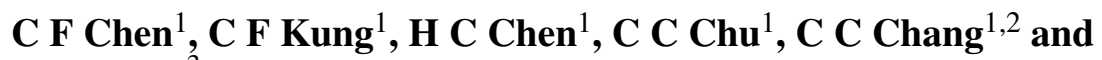 \\ F G Tseng ${ }^{3}$ \\ ${ }^{1}$ Institute of Applied Mechanics, National Taiwan University, Taipei 106, Taiwan, \\ Republic of China \\ ${ }^{2}$ Division of Mechanics, Research Center for Applied Science, Academia Sinica, Taipei 115, \\ Taiwan, Republic of China \\ ${ }^{3}$ Department of Engineering \& System Science, National Tsing-Hua University, \\ Hsinchu 300, Taiwan, Republic of China \\ E-mail: chucc@iam.ntu.edu.tw and mechang@gate.sinica.edu.tw
}

Received 14 March 2006, in final form 19 April 2006

Published 26 May 2006

Online at stacks.iop.org/JMM/16/1358

\begin{abstract}
It is known that surface tension-capillary pumping is an effective driving force in a microchannel, however a power-free mixer that uses only surface tension has not yet been achieved. In the present study, a power-free method is explored to perform mixing in a microchannel without any external active mechanisms such as pumps, valves or external energies like electrostatic or magnetic fields. The mixer is cost effective as the channel is designed to have no sidewalls with the liquid being confined to flow between a bottom hydrophilic stripe and a top-covered hydrophobic substrate. It is found from both theoretical analysis and experiments that for a given channel width, the flow rate solely due to capillary pumping can be maximized at an optimal channel height. The flow rate is in the order of nanoliters per second, for example, the flow rate is $0.65 \mathrm{~nL} \mathrm{~s}^{-1}$ at the optimal channel height $13 \mu \mathrm{m}$, given the channel width $100 \mu \mathrm{m}$. It is most crucial to this power-free mixing device that two liquid species must be well mixed before the liquids are transported to exit to a reservoir. For this purpose, asymmetric staggered grooved cavities are optimally arranged on the bottom substrate of the channel to help mixing two different liquid species. It is shown that maximum mixing occurs when the depth of the grooved structures is about two-thirds of the total channel height.
\end{abstract}

(Some figures in this article are in colour only in the electronic version)

\section{Introduction}

In the past few years, many research efforts have been directed toward developing an effective means to drive, guide and mix multiple fluid species in a microchannel. A useful microfabricated system requires mixing for initiation and activation of a physical or chemical process, such as in drug delivery $[1,2]$, biochemical synthesis $[3,4]$, premixing in fuel cells $[5,6]$. Miniaturized systems have better performance if the multi-fluidic system can be driven efficiently and the different species can be mixed rapidly in the course of fluid transport [7-10].

It has been difficult to miniaturize liquid mixers on the micro-scale due to the need of external energy resources. Most of these methods employ either actuators such as pumps or valves to provide a pressure gradient or external resources to provide thermal energy [11] or to produce electrostatic or magnetic fields $[12,13]$ to drive the fluidic inside the microchannel. Often a relatively large support is needed for the desired actuation, thus limiting the capability of system 
miniaturization and integration. In the literature, there are passive methods [14-16] and active methods [17, 18] proposed to enhance mixing in a microchannel. Active methods require external devices for improving mixing, while passive methods for mixing need no energy input but may still require a pump or pressure head to drive the fluids through the channel.

In the present study, the capillarity force is the only mechanism for driving the fluids through the microchannel. Being proportional to the contact length, the capillarity force employed as the driving mechanism on the microscale is advantageous to many other forces. It is clear that pressure force scales down quadratically and the inertia force decreases cubically with decreasing linear dimension. According to [19, 20], magnetic forces scale down from quadratically to quartically and the electrostatic forces scale down from linearly to cubically with decreasing linear dimension. Moreover, unlike magnetic or electrostatic forces which must be applied externally, capillarity is a surface property and is ideal for pumping fluids passively. However, previous applications [11, 12, 21, 22] of capillarity force in micro-fabrication require external mechanisms for controlling the capillarity. For example, the external mechanism could be thermal-capillary [11], electromagnetic [12], electro-kinetic [21] or a syringe pump [22].

The key concept of making sole use of the surface tension force is to increase the surface-to-volume ratio by reducing the depth of the channel. But the reduction cannot be continued ad infinitum as the viscous frictional force also increases. Another important concept is the reduction of the flow resistance between the fluid and the walls of the channel. They can be achieved by fabricating the microchannel to have no sidewalls; the idea was proposed sometime ago by the present authors [23, 24] and others [22, 25]. Figure 1 shows the current mixer for microchannels. Unlike in [7] where the liquid flow is confined by two-sided hydrophilic stripes, the flow channel here is defined by a bottom hydrophilic stripe (silicon dioxide) deposited on a hydrophobic substrate (Teflon) and a fully topcovered hydrophobic substrate, leaving both sides in direct contact with air. The design is cost effective because we do not have to use sidewalls, and there is no need to align hydrophilic stripes on the opposite substrates (as there is only one hydrophilic stripe on the bottom substrate). Once the fluid is pipetted into the entrance, the capillary pumping generated by the liquid meniscus at the hydrophilic interface will drag the fluid into the microchannel. It is remarked here that the top cover cannot be entirely hydrophilic because then once the fluid is introduced to the entrance, it will spread over and fill in the space between the top and the bottom substrates.

\section{Basic principle}

\subsection{Theory for a capillary pump without cavities}

For straight microchannels, Bouaidat et al [25] recently proposed an approximate theory to model the motion of the viscous liquid meniscus driven by capillarity. They made several key approximations for the majority of flow which is measured far away from the entrance and end regions:

(i) the lateral cross-section (of width $w$ and height $h$ ) of the liquid column is rectangular,
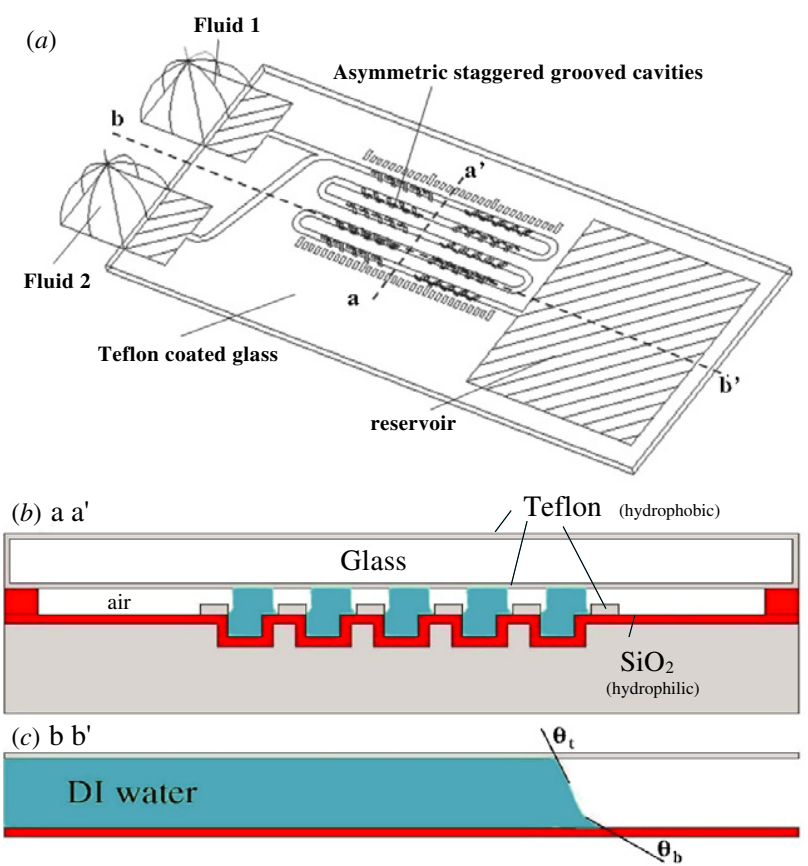

Figure 1. The design of the microchannel. The flow channel is defined by a bottom hydrophilic $\left(\mathrm{SiO}_{2}\right)$ stripe deposited on a hydrophobic substrate and a fully top-covered hydrophobic substrate (Teflon), leaving both sides in direct contact with air. Besides, in order to increase the mixing efficiency, asymmetric staggered grooved cavities are arranged at the bottom of the channel to twist liquids to produce three-dimensional flow automatically. The cutting lines $\mathrm{aa}^{\prime}$ and $\mathrm{bb}^{\prime}$ are cross-sections perpendicular and parallel to the microchannels. (a) The schematic of the device and the sideview from the cutting lines $\mathrm{aa}^{\prime}$ and $\mathrm{bb}^{\prime}$ are shown in $(b)$ and $(c) . \theta_{t}$ is the top contact angle and $\theta_{b}$ is the bottom contact angle (not to scale).

(ii) the area of the front meniscus is independent of the position of the meniscus,

(iii) the viscous flow behaviors can be described by the Poiseuille flow with a uniform shear stress $\tau \approx 6 \mu \bar{v} / h$ where $\mu$ is the viscosity of the liquid and $\bar{v}$ is the average flow velocity, and

(iv) the average flow velocity $\bar{v}$ is equal to the speed of the meniscus $\dot{x}$ where $x$ is the distance measured downstream from the entrance.

In addition, let $\rho$ denote the density of the fluid, $\mu$ the dynamic viscosity, $\gamma$ the surface tension and $\theta_{b}$ and $\theta_{t}$ the contact angles at the bottom and top interfaces, respectively. Then the equation of motion for the meniscus for $\alpha \equiv x^{2}$ is

$$
\ddot{\alpha}+A \dot{\alpha}-B=0 .
$$

Here, we have two-dimensional constants

$A \equiv \frac{12 \mu}{\rho h^{2}} \quad$ and $\quad B \equiv \frac{2\left[w\left(\cos \theta_{b}+\cos \theta_{t}\right)-2 h\right] \gamma}{\rho h w}$,

which are, respectively, the viscosity-related coefficient and the capillarity-related coefficient. By imposing the initial conditions $x(0)=0$ and $\dot{x}(0)=0$ on equation (1), the temporal evolution of the meniscus is given by

$$
x(t) \equiv \sqrt{\alpha}=\sqrt{\frac{B}{A^{2}}\left(A t+\mathrm{e}^{-A t}-1\right)} .
$$


The speed of the meniscus is readily obtained by differentiating the result with respect to time $t$,

$$
\dot{x}(t)=\frac{B}{2 A}\left(1-\mathrm{e}^{-A t}\right) / \sqrt{\frac{B}{A^{2}}\left(A t+\mathrm{e}^{-A t}-1\right)} .
$$

Equation (4) can be used to optimize the channel height for a given channel width. First of all, we note that the relaxation time $\tau \equiv A^{-1}=\rho h^{2} / 12 \mu$ is small relative to the timescale of the flow passage. Take, for example, the channel height $h=10 \mu \mathrm{m}$ with the density of water $1.0 \mathrm{~g} \mathrm{~cm}^{-3}$, its dynamic viscosity $0.01 \mathrm{~g} \mathrm{~cm}^{-1} \mathrm{~s}^{-1}$ at the room temperature $20^{\circ} \mathrm{C}$. The relaxation time $\tau$ is about $8.3 \times 10^{-6} \mathrm{~s}$, which is negligibly small compared to the time of flow passing through the channel on the order of several seconds. Therefore, equation (4) can be readily simplified to

$$
\dot{x}(t) \approx \frac{1}{2} \sqrt{\frac{B}{A(t-\tau)}} \approx \frac{1}{2} \sqrt{\frac{B}{A t}}=\frac{C}{\sqrt{t}} \quad(t \gg \tau),
$$

where

$$
C \equiv \sqrt{\frac{B}{4 A}}=\sqrt{\frac{\gamma h}{24 \mu w}\left[w\left(\cos \theta_{b}+\cos \theta_{t}\right)-2 h\right]} .
$$

Equation (5) shows that the speed of the meniscus $\dot{x}(t)$ is inversely proportional to the square root of the time with the proportional constant $C$. One immediate consequence of the expression for $C$ is that it places an upper limit for the capillary flow by requiring positivity of the term inside the square root, that is,

$$
h_{\text {upper }}=\frac{w}{2}\left(\cos \theta_{b}+\cos \theta_{t}\right) \text {. }
$$

Moreover, maximizing the flow speed for a given time $t$ is equivalent to optimizing the constant $C$ by differentiating equation (6) with respect to the channel height $h$; this gives

$$
C_{\max }=\sqrt{\frac{\gamma w}{192 \mu}}\left|\cos \theta_{b}+\cos \theta_{t}\right|
$$

at the optimal channel height

$$
h_{\mathrm{opt}}=\frac{w}{4}\left(\cos \theta_{b}+\cos \theta_{t}\right) .
$$

It is interesting to note that the optimal channel height $h_{\text {opt }}$ is exactly one-half of the upper limit of the channel height $h_{\text {upper }}, h_{\text {opt }}=\frac{1}{2} h_{\text {upper }}$.

Figure 2 shows a plot of the proportional constant $C$ versus the channel height $h$ with different settings of the channel width $w$. In the present study, the channel is $l=2 \mathrm{~cm}$ long and $w=100 \mu \mathrm{m}$ wide, and the average contact angles for the top hydrophobic and bottom hydrophilic interfaces are about $110^{\circ}$ and $30^{\circ}$, respectively. The upper limit for the channel height for capillarity pumping is $h_{\text {upper }} \approx 26 \mu \mathrm{m}$ and the optimal channel height $h_{\mathrm{opt}} \approx 13 \mu \mathrm{m}$. Under this optimal condition, the flow rate $Q=w h \bar{v}$ is about $0.65 \mathrm{~nL} \mathrm{~s}^{-1}$ where we have used the average speed $\bar{v}=500 \mu \mathrm{m} \mathrm{s}^{-1}$.

In order to measure the velocity, the frames in the video at different times are taken, and the location of the liquid front is measured. We can then calculate the velocity along the microchannel.

The theoretical result is confirmed by experiments by measuring the velocity profile versus time for different channel heights. Figure 3 shows that the maximum flow rate of deionization (DI) water driven by capillary pumping occurs

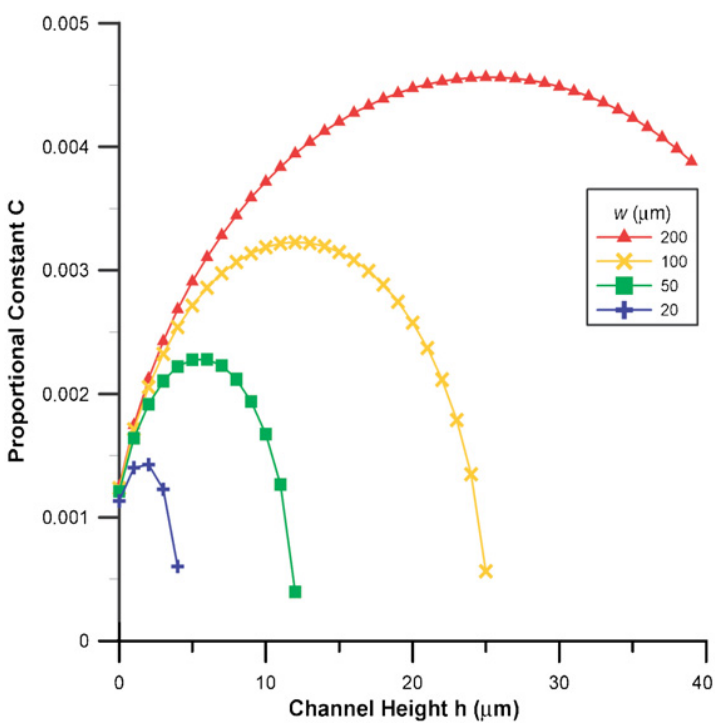

Figure 2. The proportional constants at various values of the channel width and channel height, where the contact angles for the top hydrophobic and bottom hydrophilic interfaces are about $110^{\circ}$ and $30^{\circ}$, respectively.

at $h_{\mathrm{opt}}=13 \mu \mathrm{m}$ in the range from 3 to $21 \mu \mathrm{m}$; this is in very close agreement with the theoretical analysis. It is noted that the measured speed of the meniscus from experiments under the optimal condition becomes smaller compared to the theoretical result from time $t=4 \mathrm{~s}$. This is reasonable because the flow in experiments should slow down as the flow makes its first turn at about $t=4 \mathrm{~s}$, while the theoretical analysis is valid for straight channels.

\subsection{Enhanced wave motions and mixing by asymmetric staggered grooved cavities}

The idea of using a staggered herringbone mixer or grooved mixer was first introduced by Stroock et al [7-10]. The staggered herringbone mixer can generate three-dimensional helical recirculation and mix the fluids efficiently in the microchannel. Theoretically, they derive a simple analytical expression to interpret the mixing mechanism for a pressuredriven flow over oblique grooved surfaces. The flow introduces a net flux with a transverse component, and a helical recirculation follows owing to the constraint by the sidewall boundaries.

In the present study, hydrophilic asymmetric staggered grooved cavities are etched out of the bottom substrate in order to generate significant three-dimensional mixing of the fluids by enhanced wave motions. Figure 4 shows the length scales of the present design of the microchannel. The channel height is denoted by $h$, the width by $w$, the total channel length by $l$, the depth of the cavities by $d$, the length of the cavities along the channel by $e$, the spacing between the two neighboring cavities on the same side by $s$ and the spacing between the two successive staggered grooved cavities by $s_{1}$ on the opposite sides of the channel. Here, the edges of the cavities are oblique to the main flow direction; the sloping angles of the cavities on the two opposite sides are denoted by $\alpha$ and $\beta$, respectively. In addition, there are lengths transverse 
(a)

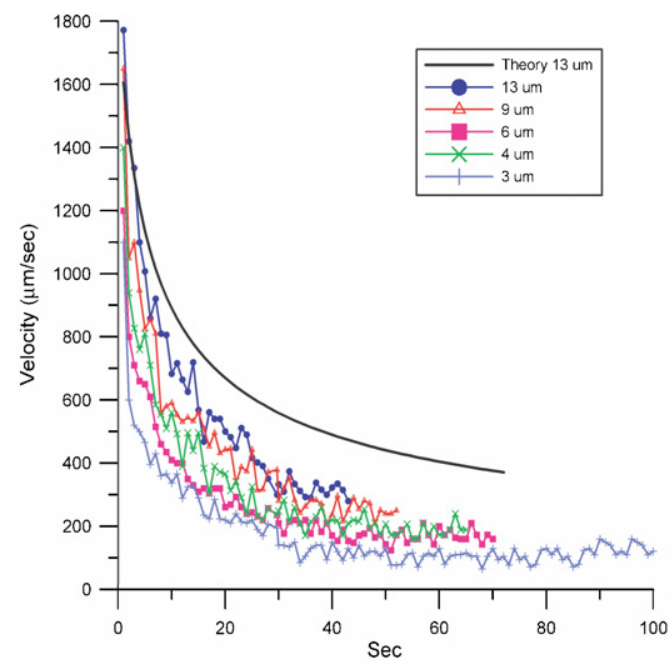

(b)

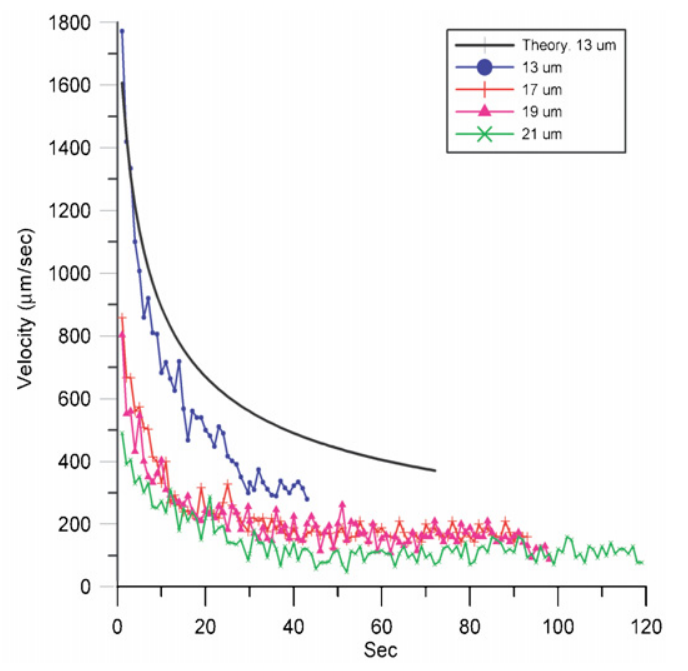

Figure 3. A comparison of the velocity for different channel heights with fixed channel width $w=100 \mu \mathrm{m}$. The contact angles for the top hydrophobic and bottom hydrophilic interfaces are about $110^{\circ}$ and $30^{\circ}$, respectively.

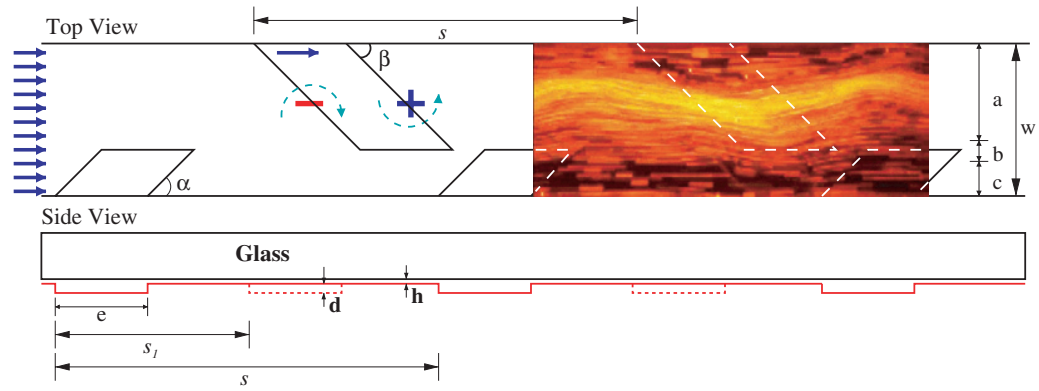

Figure 4. The various geometric parameters of the microchannel, where the channel height is denoted by $h$, the channel width by $w$, the depth of the cavities by $d$, the length of the cavities along the channel by $e$, the spacing between two neighboring cavities on the side by $s$ and the spacing between two successive staggered grooved cavities by $s_{1}$ on the opposite sides of the channel. In addition, $a, b$ and $c$ are the length scales transverse to the channel and $\alpha$ and $\beta$ denote the sloping angles of the cavities on two opposite sides, respectively.

to the channel $a, b$ and $c$, where $a$ and $c$ are, respectively, the transverse widths of the cavities on the different sides of the channel and $b=w-a-c$ is the clearance for the liquids passing between the cavities on the opposite sides.

In view of the theory of section 2.1, the total channel height $h_{\text {tot }}=h+d$ should not exceed the optimal height $h_{\text {opt }}$, and it is noted that for the design of a power-free mixer, we must choose shallower channels $(h \ll w)$ to have strong capillarity to drive the flow and deeper cavities $(d>h)$ to introduce transverse mixing by generating shear instabilities. The cavity depth $d$ cannot be too large nor too small relative to the channel height $h$. Figure 5 shows a comparison of the advancing front at different depth $d$ of the cavities. As the fluid passes over the cavity, the surface tension first drags the fluid downward. If the cavity is too deep, the fluid would experience a channel of larger height and cannot pass through it. If the cavity depth $d$ is less than a critical value, the surface tension will be enough to drag the fluid downward to the bottom and continue the motion of the liquid; however, the cavities cannot be too shallow because then there is little effect in mixing enhancement. Each encounter of two liquid species over a cavity causes a swirl in the cavity which in turn twists the flow in the main channel, and after many

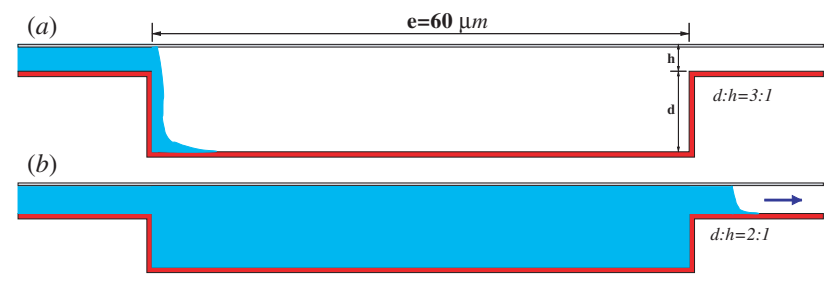

Figure 5. A comparison of the advancing front at different depths of cavities: (a) $d: h=3: 1$ and $(b) d: h=2: 1$.

twists, this eventually leads to the development of threedimensional shear flow instabilities in the flow. As shown in figure 5, when the fluids pass over the cavities, the oblique orientation of the cavity to the main stream generates shear flow (vorticity) with alternative signs along the channel. The flow will eventually become a significant wavy motion with the wavelength approximately equal to the spacing between the neighboring cavities $s$. Ideally, the asymmetric cavities on the opposite sides can generate two parallel, in-phase wave motions; these in-phase wave motions are now achieved by placing the cavities in the midst of the neighboring cavities on the opposite sides. 


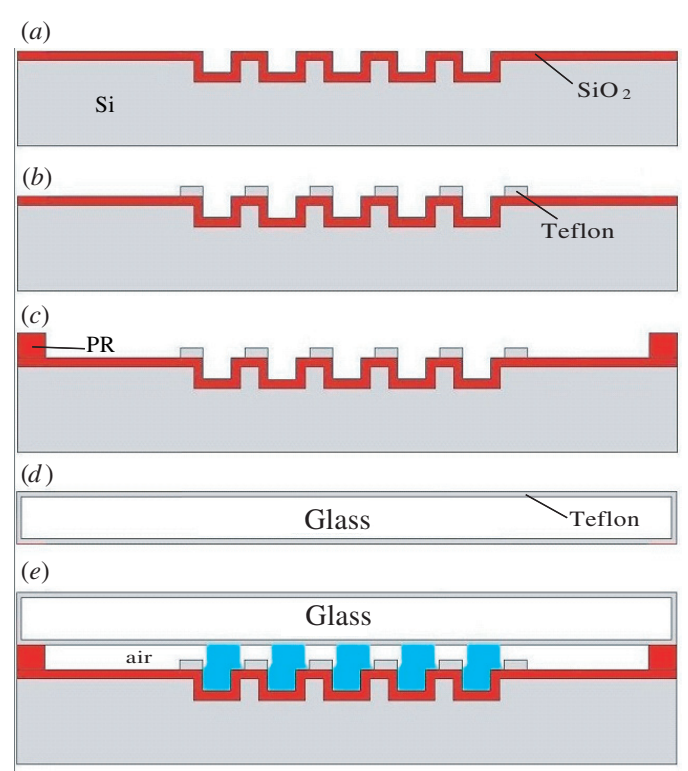

Figure 6. Fabrication process. (a) Asymmetric staggered grooved cavities defined by DSRIE and PECVD oxide $1 \mu \mathrm{m}$ coating, (b) $0.5 \mu \mathrm{m}$ Teflon coating and plasma etching, (c) PR (SJR5740) $10 \mu \mathrm{m}$ patterned as spacer, $(d)$ top glass coating on each side and (e) bounding top glass and substrate (not to scale).

\subsection{Fabrication procedure}

All these concepts put together entail the following fabrication procedure, as shown in figure 6. (a) Fabrication proceeds with etching the silicon substrate by deep silicon reactive ion etch (DSRIE) for the depth of the mixer cavities to form the asymmetric staggered grooved structures. Then, plasma enhanced chemical vapor deposition (PECVD) is used to deposit $1 \mu \mathrm{m}$ oxide for hydrophilic surface property. (b) $1 \%$ liquid Teflon (Dupont, USA) is spun on the oxide surface for $0.5 \mu \mathrm{m}$ and patterned to form a hydrophobic area. The surface of Teflon is treated with $\mathrm{O}_{2}$ plasma, then coated with hexamethyldisilazane (HMDS) to enhance adhesion of the photoresist (S1813). The photoresist is patterned with lithography and then dry etched with $\mathrm{CF}_{4}$ and $\mathrm{O}_{2}$ plasmas to form the Teflon pattern. Pattern Teflon on silicon wafer with $\mathrm{SiO}_{2}$ coating as the bottom hydrophilic channel guide, and the cover glass slide coated with Teflon as a hydrophobic liquid separation layer. (c) The process is followed by photoresist (SJR5740) patterning on the silicon substrate as a spacer for channel height definition. (d) Teflon is coated on each side of the glass substrate as the channel cover. (e) Finally, the top and bottom substrates are bonded by photoresist with the desired clearance; the plasma treated bottom surface showed hydrophilic in the channel region with $\mathrm{SiO}_{2}$ coating, but hydrophobic at the rest area with Teflon and resist coating so that the liquid can stay inside the hydrophilic channel area once dropped in.

\section{Results and discussion}

\subsection{Effect of asymmetric staggered grooved cavities}

For a useful comparison, we consider two cases that distinguish the microchannel with or without asymmetric staggered

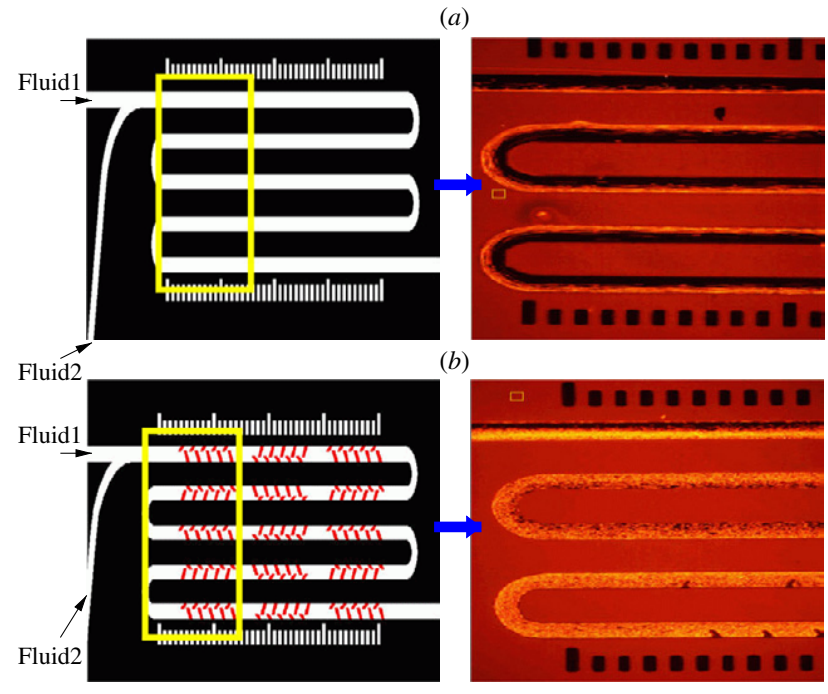

Figure 7. Fluidic mixing effect in different designs: $(a)$ a smooth channel and $(b)$ a channel with asymmetric staggered grooved structures. It is noted that red fluorescent particles were added into DI water to form the working fluids. Thus, the working fluid 1 (dark) and fluid 2 (bright) are used by purely DI water and DI water with fluorescent particles, respectively.

grooved cavities. In order to observe the mixing process, DI water and fluorescent DI water were pipetted into the microchannel. The fluorescent DI water was prepared by adding red fluorescent spheres (R900, Duke Scientific Corp.) into DI water. The concentration is $1 \% w / w$ and the diameter of spheres is $0.93 \mu \mathrm{m}$. The two liquid species were introduced into the entrance of the microchannel of $100 \mu \mathrm{m}$ width and $2 \mathrm{~cm}$ length and transported to the end of the microchannel without any driving mechanisms other than the surface tension force. The mixing process is visualized by a cooling CCD camera (DP50, OLYMPUS) under a microscope (BX60M, OLYMPUS). The mixing rate in the microchannel is analyzed by an imaging technique that measures the proportion $A \%$ of the area occupied by the fluorescent particles at different locations along the microchannel. The mixing efficiency of the device is defined to be

$$
Z=(1-|A-50| / 50) \times 100 \% \text {. }
$$

Figure 7(a) shows the flow field in the smooth channel design, where the flow is essentially a laminar flow till the end of the channel. The mixing of the two working liquids is not significant, being only through molecular diffusion across the interface of the two liquid species. Figure $7(b)$, on the other hand, shows that the design with asymmetric staggered grooved structures has significant mixing between the two liquid species in the microchannel.

\subsection{Controlling parameters of mixing efficiency}

In order to identify the major factors that affect the mixing, we shall vary (i) the height ratio $d: h$, (ii) the sloping angles $\alpha, \beta$ and (iii) the transverse length ratio $a: b: c$ with the fixed channel height $h=3 \mu \mathrm{m}$, width $w=100 \mu \mathrm{m}$, the side length of the cavities along the channel $e=60 \mu \mathrm{m}$, the spacing $s=250 \mu \mathrm{m}$ of the neighboring cavities on the same side and the spacing $s_{1}=125 \mu \mathrm{m}$ of the alternating cavities on the opposite side. 


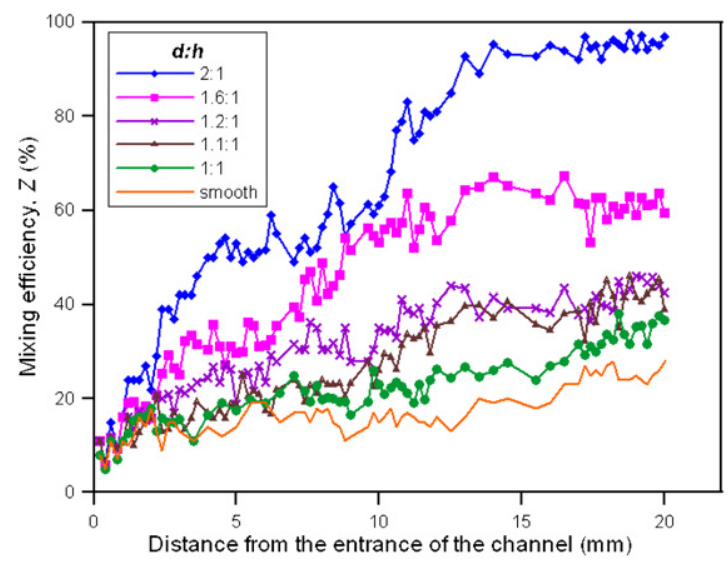

Figure 8. A comparison of the mixing rate for different height ratios $d: h$ with fixed channel height $h=3 \mu \mathrm{m}$ and width $w=100 \mu \mathrm{m}$; sloping angles $\alpha=\beta=45^{\circ}$.

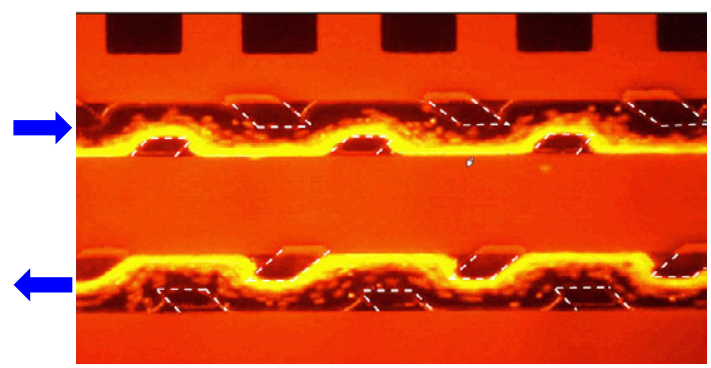

Figure 9. The flow field in deep cavity with $d: h=3: 1$.

Depth height ratio. First of all, we vary the height ratio $d: h$ from $1: 1$ to $2: 1$ with the sloping angles fixed at $\alpha=45^{\circ}$, $\beta=45^{\circ}$ and the transverse length ratio at $a: b: c=7: 0: 3$. Figure 8 shows the results of the mixing efficiency measured at different locations along the microchannel. At a smaller height ratio $d: h=1: 1$, the mixing efficiency improves a little, but becomes more significant as the ratio exceeds 1.6:1. The mixing efficiency $Z$ increases monotonically with increasing $d: h$ until it reaches $2: 1$ at which the efficiency is nearly $100 \%$, compared to $22 \%$ for the case without staggered grooved cavities at the exit of the microchannel. Beyond the critical ratio $d: h=2: 1$ the fluid flow would become laminar by forming a meandering passage in the part of the microchannel without entering the etched staggered grooved cavities (figure 9). On the other hand, the total length $h+d$ of the channel height $h$ and the cavity depth $d$ cannot exceed $13 \mu \mathrm{m}$ for the flow to pass over all the cavities. It is noted that the maximum total height $h+d$ for the channel with staggered grooved cavities would be less than that for the channel without staggered grooved cavities.

Transverse length ratio. Next, we vary the transverse length scales $a: b: c$ with the fixed height ratio $2: 1$ and the angle $\alpha=45^{\circ}\left(\beta=45^{\circ}\right)$. Figure 10 shows the results of mixing efficiency $Z$ measured at different locations along the microchannel. For the case of symmetric transverse cavity lengths $a: b: c=4: 2: 4$, the mixing efficiency already shows a better value than the case without staggered grooved cavities.

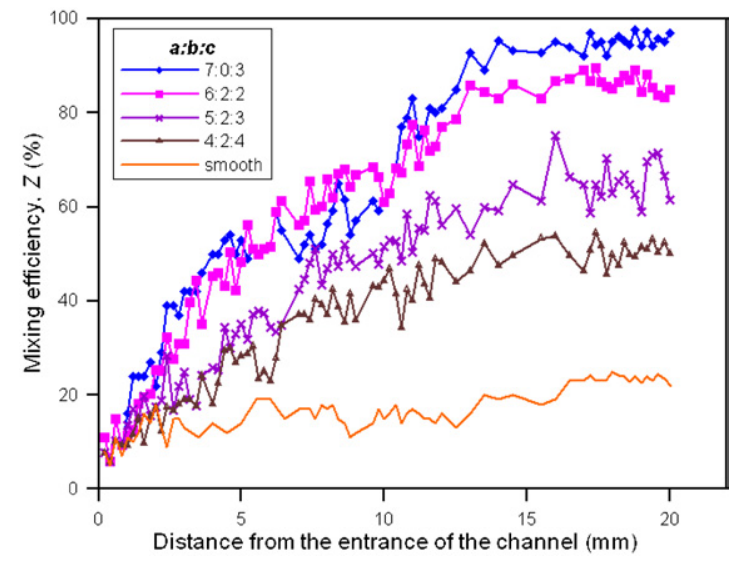

Figure 10. A comparison of the mixing rate for different transverse length ratios $a: b: c$ with fixed channel height $h=3 \mu \mathrm{m}$, width $w=$ $100 \mu \mathrm{m}$, cavity depth $d=6 \mu \mathrm{m}$ and sloping angles $\alpha=\beta=45^{\circ}$.

But the mixing efficiency can be enhanced significantly by placing asymmetric transverse lengths of cavities $(a>c)$ for which the sum $a+c$ is made large but cannot exceed the channel width $w$. The best transverse length ratio we obtain for the most conspicuous mixing is $a: b: c=7: 0: 3$ at which the clearance of a straight fluid passage $b=w-a-c$ is zero. However, it is noted that if the sum of the transverse cavity lengths $a+c$ is larger than the channel width $w$, the fluid flow would be completely blocked and there is no through-flow in the microchannel. Figure 11 shows snapshots of the flow field for different transverse length ratios $a: b: c$, captured at the location $10 \mathrm{~mm}$ downstream of the channel entrance. As we can see from these photographs, the streamlines are relatively flat at $a: b: c=4: 2: 4$ or $5: 2: 3$. But at the higher level of asymmetry with $a: b: c=6: 2: 2$ and 7:0:3, the streamlines near the interface of the two liquid species form a wavy pattern, which is enormously different from those in a channel without the design of asymmetric staggered grooved cavities. The fluid particles begin to rotate as it passes over each cavity; this twists the streamlines to form a wavy pattern. The many cavities on different sides enhance this instability by the asymmetric staggered grooved arrangement. The best arrangement of the staggered grooves should be in phase with the wavelength of instability. For example, the measured wavelength of the instability from figure $11(d)$ is about $220 \mu \mathrm{m}$ which is close to the spacing $s=250 \mu \mathrm{m}$ of the two neighboring cavities on the same side.

Sloping angle of the cavities. Another critical aspect is to examine the effect of the sloping angles $\alpha$ and $\beta$. For comparison, we fix the height ratio at $d: h=2: 1$ and the transverse length ratio at $a: b: c=7: 0: 3$. Figure 12 shows the results of mixing efficiency measured at different locations along the microchannel. It is first observed that the effect for enhancing is minimal if the cavities are placed with its transverse edges perpendicular to the mean flow direction. At the angle $\alpha=90^{\circ}\left(\beta=90^{\circ}\right)$, although the fluids enter into and stride across the cavities yet this does not produce a transverse force to affect rotation of the fluid particles. The flow field does not change much from what it is for a microchannel 

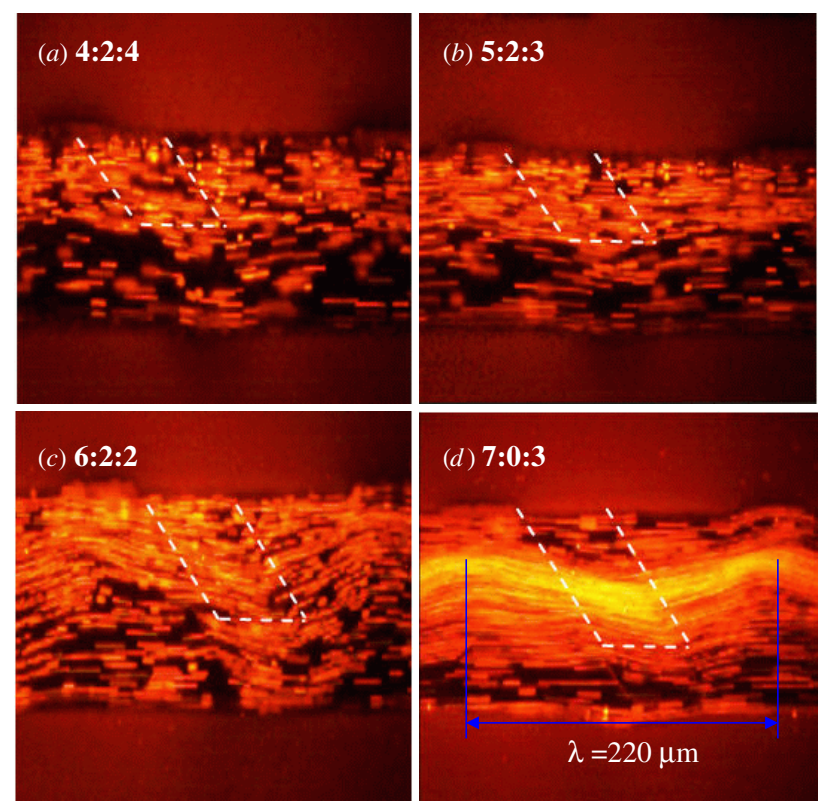

Figure 11. Snapshots of flow fields in the microchannel for different transverse length ratios $a: b: c$, while the other conditions are the same as those shown in figure 10. These pictures are captured at a location $10 \mathrm{~mm}$ downstream of the channel entrance.

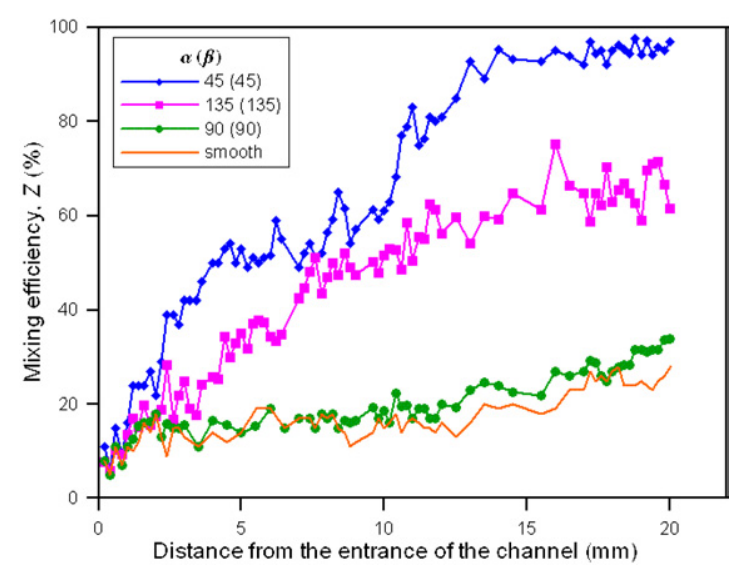

Figure 12. A comparison of the mixing rate for different angles $\alpha(\beta)$ with fixed channel height $h=3 \mu \mathrm{m}$, width $w=100 \mu \mathrm{m}$, cavity depth $d=6 \mu \mathrm{m}$ and the transverse length ratio $a: b: c=7: 0: 3$.

without asymmetric staggered grooved cavities, and remain essentially laminar. The best angle to produce a transverse force would be $\alpha=45^{\circ}\left(\beta=45^{\circ}\right)$. At these angles, the fluid particles experience a stronger shear force as they pass the edge of a cavity, and the transverse component forces the particles to rotate, twisting the streamlines and eventually leading to substantial flow instabilities after passing several cavities. Figure 13 shows the flow patterns at different stages of flow development with the optimal parameters: the height ratio at $d: h=2: 1$, the sloping angles at $\alpha=45^{\circ}\left(\beta=45^{\circ}\right)$ and the transverse length ratio at $a: b: c=7: 0: 3$. The flow is laminar at the entrance region (figure 13(a)), but then gradually turns to be vortical when passing through the first few asymmetric staggered grooved cavities as the fluid particles start to scatter (figure 13(b)). The fluids reach the stage of $45 \%$ mixing at the distance $5 \mathrm{~mm}$ downstream of the entrance (figure 13(c)), and finally reaches a stage of almost fully mixing at the distance
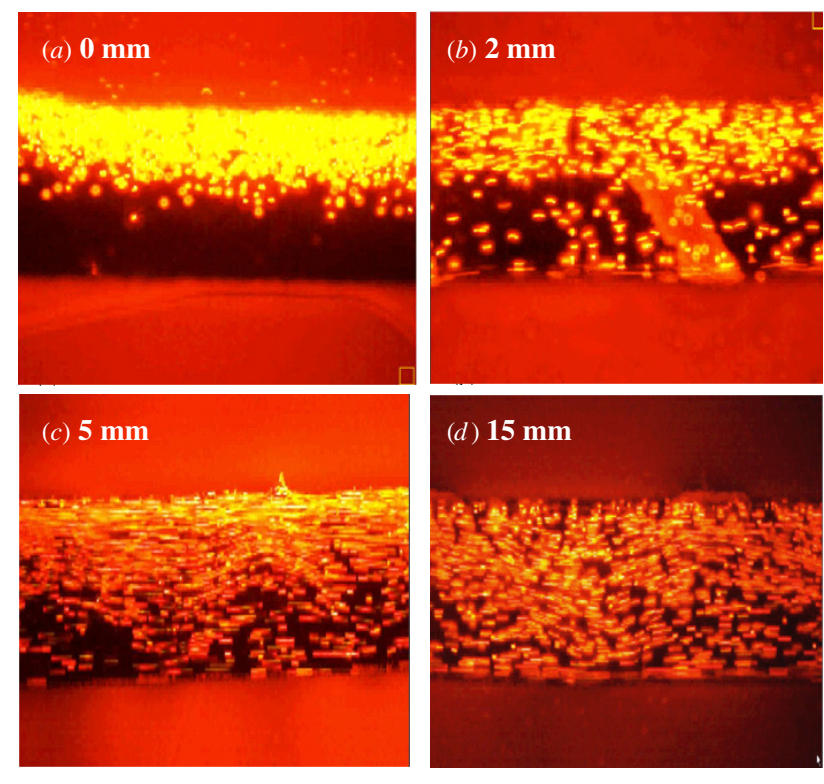

Figure 13. Snapshots of the particle distribution on different locations of the channel with asymmetric staggered grooved structures for (a) $0 \mathrm{~mm},(b) 2 \mathrm{~mm},(c) 5 \mathrm{~mm}$ and (d) $15 \mathrm{~mm}$ downstream of the entrance, respectively, where the channel height $h=3 \mu \mathrm{m}$, width $w=100 \mu \mathrm{m}$, cavity depth $d=6 \mu \mathrm{m}$ and transverse length ratios $a: b: c=7: 0: 3$ and sloping angles $\alpha=\beta=45^{\circ}$.

$15 \mathrm{~mm}$ downstream of the entrance (figure $13(d)$ ). Under these optimal specifications, the device can transport and mix the liquids very efficiently within $40 \mathrm{~s}$ from one end of the channel to another. More precisely, the volume flow rate for complete mixing is $6 \mathrm{~nL}(h \times w \times l=3 \mu \mathrm{m} \times 100 \mu \mathrm{m} \times 0.02 \mathrm{~m}, d=$ $6 \mu \mathrm{m})$ at an interval of $40 \mathrm{~s}$.

\section{Concluding remarks}

In summary, the presently proposed nanoliter mixer for microchannels has the following features. It is power free with the surface tension-capillary pumping the only mechanism for driving liquids. The microchannel is designed to have no sidewalls for reducing the frictional force, with the fluid being confined to the flow between the bottom hydrophilic stripe and the fully top-covered hydrophobic substrate. It is shown from both theoretical analysis and experiments that the optimal channel height for straight channels is one-half of the least upper limit of the channel height for capillary pumping. Flow instabilities are induced to enhance mixing by the carefully designed asymmetric staggered grooved cavities on the bottom hydrophilic substrate. The spacing of the staggered grooved structures is chosen to be approximately in phase with the wavelength of the induced flow instability for resonant magnification. In achieving full mixing at the exit of the microchannel, we found the optimal conditions if the ratio of the cavity depth to the channel height is $d: h=2: 1$, the transverse length ratio is $a: b: c=7: 0: 3$ and the sloping angle of the cavity edges is $\alpha=\beta=45^{\circ}$. The asymmetry of the transverse cavity lengths $a$ and $c$ with zero passage for straight fluid flow $b$ helps the development of flow instabilities. A sloping angle of $45^{\circ}$ is best to produce transverse shear forces to rotate the fluid particles, twisting the streamlines that 
alter the laminar flow pattern to be vortical. The device mixes liquid species at the rate of a few nanoliters within tens of seconds. For example, at the optimal ratio $d: h=6 \mu \mathrm{m}: 3 \mu \mathrm{m}$ for complete mixing, given the channel width $w=100 \mu \mathrm{m}$, the flow rate is $6 \mathrm{~nL}$ at the interval of $40 \mathrm{~s}$; this qualifies the current device as a nanoliter mixer for microchannels. All these salient features render the presently proposed capillary pumping nanoliter mixer for microchannels cost effective as the entire device is easily amenable to miniaturization by the modern fabrication technique of planar lithography.

\section{Acknowledgments}

The work was supported in part by the National Science Council of the Republic of China (Taiwan) under contracts NSC 93-2212-E-002-041 and 93-2212-E-002-037. The authors would also like to thank Professor $\mathrm{T} \mathrm{Y} \mathrm{Wu}$ of Caltech for his constant encouragement and helpful comments/ discussion on an earlier draft of the paper.

\section{References}

[1] LaVan D A, Lynn D M and Langer R 2002 Nat. Rev. Drug Discov. $177-84$

[2] Saltzman W M 2001 Drug Delivery: Engineering Principles for Drug Delivery (London: Oxford)

[3] Service R F 1998 LABS ON A CHIP: coming soon: the pocket DNA sequencer Science 282 399-401

[4] Burns M A et al 1998 An integrated nanoliter DNA analysis device Science 282 484-7

[5] Mazumder S K, Acharya K, Haynes C L, Williams R Jr, Spakovsky M R V, Nelson D J, Rancruel D F, Hartvigsen J and Gemmen R S 2004 Solid-oxide-fuel-cell performance and durability: resolution of the effects of powerconditioning systems and application loads IEEE Trans. Power Electron. 19 1263-78

[6] Karnik S V, Hatalis M K and Kothare M V 2003 Towards a Palladium micro-membrane for the water gas shift reaction: microfabrication approach and hydrogen purification results J. Microelectromech. Syst. 12 93-100

[7] Stroock A D, Dertinger S K W, Ajdari A, Mezic I and Stone H A 2002 Chaotic mixer for microchannels Science 295 647-51

[8] Stroock A D, Dertinger S K W, Whitesides G M and Ajdari A 2002 Patterning flows using grooved surfaces Anal. Chem. 74 5306-12
[9] Stroock A D and Whitesides G M 2003 Controlling flows in microchannels with patterned surface charge and topography Acc. Chem. Res. 36 597-604

[10] Stone H A, Stroock A D and Ajdari A 2004 Engineering flows in small devices: microfluidics toward a lab-on-a-chip Annu. Rev. Fluid Mech. 36 381-411

[11] Kataoka D E and Troian S M 1999 Patterning liquid flow on the microscopic scale Nature 402 794-7

[12] Yun K S, Cho I J, Bu J U, Kim C J and Yoon E 2002 A surface-tension driven micropump for low-voltage and low-power operations J. Microelectromech. Syst. $11454-61$

[13] Washizu M 1998 Electrostatic actuation of liquid droplets for microreactor application IEEE Trans. Ind. 34 732-7

[14] Koch M, Chatelain D, Evans A G R and Brunnschweiler A 1998 Two simple micromixers based on silicon J. Micromech. Microeng. 8 123-6

[15] Voldman J, Gray M L and Schmidt M A 2000 An integrated liquid mixer/valve J. Microelectromech. Syst. 9 295-302

[16] Simonnet C and Groisman A 2005 Chaotic mixing in a steady flow in a microchannel Phys. Rev. Lett. 94134501

[17] Ryu K S, Shaikh K, Goluch E, Fan Z and Liu C 2004 Micro magnetic stir-bar mixer integrated with parylene microfluidic channels Lab Chip 4 608-13

[18] Sasaki N, Kitamori T and Kim H B 2006 AC electroosmotic micromixer for chemical processing in a microchannel Lab Chip 6 550-4

[19] Trimmer W S N 1989 Microrobots and micromechanical systems Sensors Actuators 19 267-87

[20] Cho H J and Ahn C H 2002 A bidirectional magnetic microactuator using electroplated permanent magnet arrays J. Microelectromech. Syst. 11 78-84

[21] Gallardo B S, Gupta V K, Eagerton F D, Jong L I, Craig V S, Shah R R and Abbott N L 1999 Electrochemical principles for active control of liquids on submillimeter scales Science 283 57-60

[22] Zhao B, Moore J S and Beebe D J 2001 Surface-directed liquid flow inside microchannels Science 291 1023-26

[23] Chu C C, Yao L Y, Chang C C, Kuo S C and Tseng F G 2000 Surface tension driven flow inside top-bottom constrained microchannels Bull. Am. Phys. Soc. 45186

[24] Chen C F, Kuo S C, Chu C C and Tseng F G 2003 A power-free liquid driven method for micro mixing application Proc. IEEE MEMS Conf. (Kyoto, Japan) pp 100-3

[25] Bouaidat S, Hansen O, Bruus H, Berendsen C, Bau-Madsen N K, Thomsen P, Wolff A and Jonsmann J 2005 Surface-directed capillary system: theory, experiments and applications Lab Chip 5 827-36 